\title{
Pulmonary hypertension in congenital heart disease
}

\author{
Emma Pascall ${ }^{1}$ \& Robert MR Tulloh*,1 \\ ${ }^{1}$ Department of Congenital Heart Disease, Bristol Heart Institute, Upper Maudlin Street, Bristol, BS2 8BJ, UK \\ *Author for correspondence: Tel.: +44 117342 8856; Fax: +44 117342 8857; robert.tulloh@bristol.ac.uk
}

Pulmonary hypertension is defined as a mean pulmonary arterial pressure $\geq 25 \mathrm{mmHg}$. We focus on its relevance in congenital heart disease, reviewing pathophysiology, diagnosis and management. Pulmonary hypertension is a relatively common complication of congenital heart disease, with adult prevalence between 5 and $10 \%$. A multifactorial cause is recognized, relating to the size and nature of cardiac defect as well as environmental and genetic factors. More complex disease is increasingly recognized rather than pure Eisenmenger complex. Remodeling of the pulmonary vascular bed causes increased pulmonary vascular resistance diagnosed by a collection of investigations including echocardiography, exercise testing, cardiac catheterization, MRI and CT scanning. Management employs disease-modifying medications which are now used with increasing benefit.

First draft submitted: 21 June 2017; Accepted for publication: 22 February 2018; Published online: 24 May 2018

Keywords: congenital heart disease $\bullet$ Eisenmenger $\bullet$ pulmonary hypertension $\bullet$ pulmonary vascular resistance

Pulmonary hypertension (PH) is a relatively common complication of congenital heart disease (CHD), seen in about $10 \%$ of adult cases. It is defined as a mean pulmonary arterial pressure (PAPm) $\geq 25 \mathrm{mmHg}$ measured during right heart catheterization at rest. Pulmonary arterial hypertension (PAH) defines a subgroup of patients with $\mathrm{PH}$ who are diagnosed with precapillary $\mathrm{PH}$. This is confirmed by a pulmonary artery wedge pressure $\leq 15 \mathrm{mmHg}$ and a pulmonary vascular resistance (PVR) $>3$ Wood Units in patients with no other causes for precapillary PH, which would include lung pathology and chronic thromboembolic pulmonary hypertension [1].

Updated hemodynamic definitions of pulmonary hypertension and its subgroups along with the updated clinical classification are shown in Box $1[2,3]$.

This article looks at pulmonary hypertension associated with CHD; particularly focusing on the pathophysiology, diagnosis, treatment and outcomes of the condition.

\section{Epidemiology}

In the UK, the prevalence of $\mathrm{PH}$ has been reported as 97 cases per million with a female to male ratio of 1.8. Furthermore, the age-standardized death rate in the USA from PH ranges from 4.5 to 12.3 per 100,000 population [3].

Focusing on PAH associated with CHD (CHD-APAH), there is a prevalence of $5-10 \%$ of adults with $\mathrm{CHD}$ and this has a large impact on mortality and morbidity leading to increasing requirements for lifelong care [4]. A Dutch study focused on the epidemiology of patients with septal defects and they showed that CHD-APAH was seen in $6.1 \%$ of these patients with a median age of 38 years and that $60 \%$ were female $[5,6]$. The calculated incidence of CHD-APAH is 2.2 per million of the population with a prevalence of 15.6 per million, $58 \%$ of whom had Eisenmenger syndrome.

Furthermore, ventricular septal defect (VSD) was the most frequent underlying defect (42\%). This study also highlights the risk of $\mathrm{PH}$ after surgery to close defects with $3 \%$ of these patients having ongoing $\mathrm{PH}$.

\section{Causes \& pathophysiology}

The severity of CHD-APAH can vary greatly despite the presence of similar underlying cardiac lesions, highlighting the dynamic nature of the condition and its multifactorial etiology [7]. The cause of CHD-APAH is dependent on

Future : Medicine 
Box 1. Hemodynamic definitions of pulmonary hypertension and its subgroups.

1. Pre-capillary: mean PAP is $\geq 25 \mathrm{mmHg}$, but left atrial pressure (or pulmonary capillary wedge pressure) $\leq 15$ $\mathrm{mmHg}$

2. Post-capillary: mean PAP is $\geq 25 \mathrm{mmHg}$, but left atrial pressure (or pulmonary capillary wedge pressure) $>15$ $\mathrm{mmHg}$

Data taken from [3].

PAP: Pulmonary artery pressure.

the defect present but it is also thought that environmental factors and genetics or epigenetics play a role. This is illustrated by a study showing that $6 \%$ of patients with CHD-APAH were found to have mutations of the BMPR2 which is also associated with familial and idiopathic PAH but to a lesser extent (75 and 25\% respectively) [8]. This along with mutations of the gene encoding the protein, ALK-1 are well-known to cause PAH [7,9].

Pulmonary hypertension in CHD is commonly secondary to left-to-right shunt defects or left heart obstructive disease causing postcapillary hypertension [10]. Common defects include VSD, atrial septal defect (ASD) and persistent ductus arteriosus. Studies have shown the size of the defect to be influential in whether patients develop PAH [11]. For example, the natural history of patients with VSDs shows that of those with small or moderate defects $(\leq 1.5 \mathrm{~cm}$ in diameter), $3 \%$ of patients will develop Eisenmenger syndrome. However, in large defects $(>1.5 \mathrm{~cm})$ all develop the syndrome in time, if there is no intervention. For ASDs, a recent publication demonstrates the same finding, that defects most likely to have severe PAH were the largest $(31.84 \pm 8.21 \mathrm{~mm})$ [12]. More complex lesions such as atrioventricular septal defects or truncus arteriosus often develop PAH early in life. Furthermore, patients can be diagnosed with PAH after correction of the initial heart defect. It is unclear whether this is due to progression of the pulmonary vascular disease despite surgical correction but data shows that early correction acts to help prevent subsequent development of PAH [13]. Specifically, there has been much interest in PH after the Fontan operation. This operation is the definitive surgery in staged palliation for children born with single-ventricle CHD [14]. There have been huge improvements with early outcomes through early operative success over the past two decades, however there is still late mortality and morbidity associated with pulmonary hypertension. The lack of a subpulmonary ventricle to push blood through the pulmonary vasculature leads to reduced cardiac filling and hence reduced cardiac output [15]. In the long term, there is increased PVR to blood flow through the pulmonary system, without the pressures being as high as a mean of $25 \mathrm{mmHg}$. The pathophysiology is different, resulting from a complex series of interactions: lack of pulsatile flow, poor respiratory mechanics in addition to reduced systemic ventricle diastolic, long axis and systolic function.

For large, nonrestrictive ventricular or arterial communications, the resistance difference between the systemic and pulmonary circulation will largely determine the size of the shunt and its direction [11]. However, in patients with a large atrial communication, a right-to-left shunt can be seen in association with systemic or suprasystemic pulmonary arterial pressures (PAP). The shunt may reflect a decreased compliance of the right ventricle due to ventricular hypertrophy or fibrosis. Furthermore, atrial connections, even with large defects, develop Eisenmenger much later in life than in defects at other levels [7,16]. Pretricuspid shunts are low-pressure left-to-right shunts initially causing volume overload of the right ventricle. Thus, volume overload of the pulmonary circulation occurs but the low-pressure system does not result in an immediate increase in PAP. The risk of developing PAH is mostly determined by the size of the ASD but also the compliance of the right ventricle [17]. However, left heart lesions along with left ventricular dysfunction also play a role. Overall, pretricuspid lesions rarely develop Eisenmenger, illustrated by the fact that only $2 \%$ of patients with ASD develop Eisenmenger.

Post-tricuspid lesions are high-pressure left-to-right shunts leading to volume overload on the left ventricle and pulmonary circulation. In these defects, the development of PAH occurs in the first years of life. If this is left untreated, almost all will develop shunt reversal through the development of suprasystemic PVR, namely the Eisenmenger complex.

The pathophysiology of PAH in Eisenmenger relates to vasoconstriction, medial wall hypertrophy and remodeling of the pulmonary vascular bed [17]. Histology of the pulmonary arteries in CHD-APAH shows extension of smooth muscle cells into peripheral pulmonary arteries, medial hypertrophy, intimal proliferation fibrosis, plexiform lesions and rarefication of the pulmonary arterial tree [18]. The hypothesis for mechanism of damage is that high flow and pressure causes pulmonary vascular endothelial damage with the destruction of endothelial barrier function. This then causes activation of vascular elastase and matrix metalloproteinases, leading to degradation of the extracellular 
matrix and release of FGF and TGF- $\beta 1$. Such release leads to smooth muscle cell hypertrophy and proliferation and neo-intima formation [19]. Inflammation and thrombosis is thought to occur through the adherence and activation of platelets and leukocytes after endothelial damage with secondary activation of the coagulation pathways. Overall, the endothelial dysfunction and hence vascular remodeling of the pulmonary arteries leads to increased PVR and eventual right ventricular failure [20].

The endothelial dysfunction causes an increase in vasoconstrictors (endothelin-1 and thromboxane) and decrease in vasodilators (nitric oxide, vasoactive peptide and prostaglandin $\mathrm{I}_{2}$ ) [17]. It is through this vasoconstriction that vascular remodeling occurs [21]. Evidence for this comes from studies showing circulating endothelin levels correlate with disease severity and outcome in patients with PAH [22]. Furthermore, decreased levels of prostacyclin, a potent pulmonary and systemic vasodilator, have been associated with PAH through impaired production [23,24]. Emerging evidence in CHD-APAH shows increased levels of the vasoconstrictor serotonin compared with healthy controls and altered intrapulmonary expression of TGF- $\beta 1$ its receptors [25].

There are different ways to classify CHD-APAH but a recent classification is commonly used [17].

\section{Presentation \& diagnosis}

The management of pulmonary hypertension in CHD relies on correctly identifying clinical signs and symptoms as well as performing investigations via imaging and blood tests, in order to reach a diagnosis based on hemodynamic criteria as already discussed; while identifying the cause and hence appropriate treatment.

\section{Clinical signs \& symptoms}

Initial symptoms are typically induced by exercise and related to progressive right ventricular dysfunction. Shortness of breath, fatigue, angina and syncope are seen. Most adults with Eisenmenger complex will have exercise intolerance with studies showing $>90 \%$ of patients are in WHO class II or worse and 50\% report severe limitations [21]. In more advanced cases, symptoms at rest are present. Additionally, as right heart failure progresses classical signs of abdominal distension and ankle edema may be seen.

Less commonly, symptoms can relate to the abnormal blood flow in the pulmonary vascular bed such as hemoptysis relating to rupture of the hypertrophied bronchial arteries, or hoarseness due to compression of the left recurrent laryngeal nerve associated with dilated pulmonary artery [3]. Wheeze is present when there is compression of the large airways and angina when there is compression of the left main coronary artery between the dilated pulmonary artery and the aorta.

Clinical signs of pulmonary hypertension include left parasternal lift, loud second heart sound, a third heart sound related to the right ventricle, pansystolic murmur (tricuspid regurgitation) and diastolic murmur (pulmonary regurgitation) [3]. If $\mathrm{PAH}$ is advanced, raised jugular venous pressure, hepatomegaly, ascites, peripheral edema and cool peripheries may be seen. Patients are often cyanosed, and so it is important to record the presence of clubbing, hepatic and renal dysfunction, ischemic complications and the stigmata of endocarditis.

\section{Investigations}

Basic investigations should first be carried out including electrocardiography (ECG), chest radiography, peripheral oxygen saturations, objective assessment of exercise tolerance and echocardiography. Additional information of the pulmonary vascular bed and right ventricle can then be assessed with chest computed tomography (CT) and MRI.

\section{Electrocardiography}

It should be highlighted that, although an electrocardiogram can demonstrate evidence for PH, a normal ECG does not exclude the diagnosis. Findings related to CHD-APAH can include right atrial hypertrophy, right axis deviation, right bundle branch block, right ventricular strain and hypertrophy [3]. The latter can be calculated by the sum of the R-wave amplitude in V1 and maximum amplitude of the S wave in V5 or V6. Evidence suggests this may provide prognostic detail in patients with Eisenmenger complex [26]. Studies have shown RV strain is more sensitive in demonstrating PH than RV hypertrophy [27]. This is especially true in patients with complex CHD, where the mean frontal QRS axis may be rightward even under usual circumstances.

\section{Chest radiography}

Findings from chest radiography in patients with CHD-APAH include pulmonary artery dilatation, aneurysms or calcification [17]. There may also be right atrial and/or right ventricular enlargement and the cardiothoracic ratio 
can be measured. In patients with left heart disease, pulmonary venous congestion can be seen. Moreover, there may be consolidation related to pulmonary hemorrhage or infiltrates. It should be noted though that many radiographs can be normal in these patients.

\section{Exercise testing}

This can be done with either a 6-min walk test or cardiopulmonary exercise testing with measurement of peak oxygen consumption. Both are routinely used in assessment of CHD-APAH and a reduction of distance in the former and peak oxygen in the latter have been associated with impaired prognosis in these patients [28,29] In addition, evidence of desaturation on exercise can imply that there might be reversal of shunt and evidence of potential right to left shunting in ASDs as evidence of possible raised PVR and helps to predict survival [30].

\section{Laboratory tests}

These should include full blood count, urea and electrolytes, liver function tests and uric acid (the latter is often high due to breakdown of increased levels of hemoglobin). The hemoglobin should be focused on as well as iron levels as shown by transferrin saturation and serum ferritin. The importance of this is demonstrated by studies showing consumption of iron stores due to excessive erythropoiesis in CHD-APAH [31]. However, it must be noted that in patients with cyanotic CHD classical microcytosis and hypochromia only occurs in around $16 \%$ of patients. Instead, due to coexistent folate or vitamin B12 deficiency, a hyperchromia and/or macrocytosis picture is seen. Last, patients with Eisenmenger syndrome commonly have iron deficiency anemia that is thought to be multifactorial in origin. In addition, it has been shown that iron deficiency leads to a worse outcome [32]. For this reason, venesection is strongly discouraged as there is an increased risk of stroke and to avoid iron deficiency.

\section{Genetic testing}

Genetic analysis should also be performed in many patients where there is a suspected association between the patient's signs and symptoms and a genetic abnormality. Furthermore, where there is a family history of pulmonary hypertension or discrepancy between the signs and symptoms relating to $\mathrm{PH}$ and underlying CHD, testing should look for alternative causes such as idiopathic or familial PH. Genetic counselling and BMPR2 mutation screening (point mutations and large rearrangements) can be offered in these cases [9]. Screening for rarer mutations are offered in certain patients.

\section{Echocardiography}

Transthoracic echocardiography will allow visualization of underlying cardiac anatomy, levels of shunting and ventricular function. Furthermore, it can be used to estimate subpulmonary ventricular pressure and highlights direction and gradient of flow across shunts. Doppler measurements can be used to estimate PAP. Systolic PAP is estimated with peak tricuspid regurgitation velocity and adding right atrial pressure. The simplified Bernoulli equation $\mathrm{P}=4 \mathrm{~V}_{2}{ }^{2}+\mathrm{RA}$ pressure is used where $\mathrm{P}$ is estimated pulmonary artery systolic pressure $(\mathrm{mmHg}), \mathrm{V}_{1}$ is peak Doppler estimated velocity across the tricuspid valve $(\mathrm{m} / \mathrm{s})$, provided there is no right ventricular outflow tract obstruction [3]. Echocardiography can be used to estimate right atrial pressure (normal range $0-5 \mathrm{mmHg}$ ). This is based on the diameter of the inferior vena cava and respiratory variation of this diameter. However, due to inaccuracies with this method, the most recent ESC/ERS guidelines for the diagnosis and treatment of pulmonary hypertension suggest using continuous wave Doppler measurements of peak tricuspid regurgitation velocity as the determinant for diagnosis PH through echocardiography [3]. However, in patients with severe tricuspid regurgitation, this may be underestimated and therefore caution should be used. There may also be overestimation and therefore other features suggestive of $\mathrm{PH}$ on echocardiography must be included. These include the presence of peak tricuspid regurgitation velocity. If this is less than $\leq 2.8$ or not measurable, then it is unlikely to be suggestive of $\mathrm{PH}$, but if there are other signs of $\mathrm{PH}$, then be careful of underestimating the pressure. If the tricuspid regurgitation is between 2.9-3.4, then the index of suspicion is raised. It is even more likely if tricuspid regurgitation jet velocity is $>3.4$, without any other signs of $\mathrm{PH}$ [3]. The probabilities can then be used to determine if cardiac catheterization is needed in individual patients.

The other echocardiographic 'PH signs' provide assessment of RV size and pressure overload, pulmonary artery acceleration time, and pulmonary artery diameter $[3,33]$.

If there is no detectable shunt, or significant dilatation of the proximal pulmonary artery with only moderate $\mathrm{PH}$, and high pulmonary blood flow is visualized with transthoracic echocardiogram using pulsed Doppler wave, 
a transesophageal echocardiogram with contrast or cardiac magnetic resonance (CMR) imaging may be needed to exclude sinus venosus ASD and anomalous pulmonary venous return.

Echocardiography is not always sufficient to diagnose $\mathrm{PH}$ when treatment is being considered. The standard teaching is that cardiac catheterization must be performed. However, this is not usually practical in those patients with learning difficulties such as Down's syndrome, nor is it essential if there is clear evidence of Eisenmenger syndrome, with clinical confirmation of the PVR being higher than the systemic vascular resistance. The indication for cardiac catheterization, of course, would be stronger if there was evidence that it might be possible to close or partially close the defect.

\section{Cardiac catheterization}

Right heart catheter is sometimes required to confirm the diagnosis of CHD-APAH. It is also needed to assess severity of hemodynamic impairment and carry out vasoreactivity testing. However, it should only be carried out after performing the less invasive tests described above to avoid unnecessary procedures where an alternative diagnosis is made. Additionally, it should be carried out in expert centers to ensure high-quality results with low risk to patients. It is certainly of benefit in those patients when there is evidence of multifactorial causation of the $\mathrm{PH}$. For example, there may be pulmonary venous hypertension in addition to CHD-APAH. In those patients with the Fontan circulation, accurate measurement of pulmonary artery pressure, transpulmonary gradient and PVR is mandatory before starting therapy. In these patients, the pulmonary artery pressure is always low, due to the surgical connection between the systemic veins and the pulmonary arteries. However, abnormalities of the pulmonary vascular tree can still ensue, often thought of as a reduced compliance in the circuit rather than elevated PVR.

The mean PAPm is measured by right heart catheter, and for a diagnosis of PAH to be made, PAPm should be $\geq 25 \mathrm{mmHg}$ at rest (with exception of the Fontan circuit as above) [3]. Potential vasoreactivity of the pulmonary vascular bed is assessed by cardiac catheterization while breathing $100 \%$ oxygen via rebreathing mask, inhaled nitric oxide and intravenous adenosine or prostacyclin. It has recently been shown to help determine prognosis in adults with Eisenmenger complex [34]. An absent or negative pulmonary vasoreactivity study would not impede starting disease-targeting treatment.

\section{Cardiac MRI resonance imaging}

MRI is used to clarify intracardiac anatomy and hence the location and size of intra and extracardiac communications. This can be used to give a definitive diagnosis in patients with PAH with suspected CHD if echocardiography is inconclusive. It also demonstrates the pulmonary vascular bed and can accurately give an evaluation of RV size, morphology and function. Moreover, it is a noninvasive means to assess blood flow, including cardiac output, stroke volume and pulmonary arterial distensibility [3]. Although no single CMR measurement excludes PH, highly suggestive features for the diagnosis include, reduced pulmonary arterial distensibility, retrograde flow and the presence of late gadolinium enhancement [35,36]. Modern hybrid CMR imaging, allows for measurement of right heart pressures with indwelling venous lines and MRI measurement of pulmonary blood flow. This gives very accurate measures of PVR, but is not available in all centers.

\section{CT scans}

All CT scans in cardiac centers are now high resolution and this is the modality of choice for assessing lung parenchyma, especially to exclude intrapulmonary hemorrhage or infarction. It is widely available and can be used to visualize and give information on vascular, cardiac, mediastinal and pulmonary abnormalities. Specifically, in symptomatic patients, CT scan highlights the evidence for a diagnosis of $\mathrm{pH}$ through increased PA diameter $(\geq 29 \mathrm{~mm})$ and pulmonary artery to ascending aorta diameter ratio $(\geq 1.0)$ [37]. Moreover, segmental artery to bronchus ratio $>1: 1$ in three or four lobes is highly specific for a diagnosis of $\mathrm{PH}$ [37]. In addition, CT imaging is required, often in conjunction with ventilation: perfusion imaging to rule out the additional presence of chronic thromboembolic disease, especially if it were amenable to surgical intervention.

\section{Treatment}

CHD-APAH should be managed in specialist centers, those that see CHD and patients with $\mathrm{PH}$ on a regular basis. The standards applicable to CHD-PAH centers are not yet defined, being clarified by a number of reviews in the UK and other countries. Additionally, patient education, awareness of potential risks and complications 
and behavioral modifications are vital in the management of these patients. It is recommended to avoid strenuous exercise, however mild activities are beneficial. Patients can present with clinical deterioration at any point during their clinical course. These include with dehydration, lung infections, when at high altitudes and when requiring general anesthesia for noncardiac surgery.

Pregnancy is associated with a high risk to both mother and fetus hence effective contraception is important. Due to the interaction of contraceptives with progesterone-based compounds, those taking Endothelin receptor antagonists should have dual contraception [3].

\section{Standard treatment}

Standard treatment for CHD-APAH includes diuretics for any fluid accumulation. This is a late sign and so treatment is symptomatic for hepatic congestion, ascites and peripheral edema [3]. In practice, digoxin is only used in the presence of arrhythmias. The evidence for oxygen therapy is very limited and is really restricted to those patients with low levels of oxygen saturations at night time, especially if there is airway obstruction or if there is concurrent lung disease [38].

Calcium channel blockers and anticoagulation are not advised in CHD [3]. Specifically, there is increased mortality with the use of anticoagulation therapy in CHD-APAH from hemoptysis. Nondihydropyrodine calcium channel blockers are negatively inotropic and so are not recommended.

\section{Disease-modifying treatment}

Disease modifying therapy is used to act on three pathways in the control of PVR. There is medication which currently interacts with three pathways: the nitric oxide pathway, the endothelin pathway and the prostanoid pathway. There has been increasing evidence of the efficacy of these therapies in patients with CHD-APAH [3].

ERS/ESC guidelines highlight treatment goals for children and adults as WHO functional class, tricuspid annular plane systolic excursion and $\mathrm{N}$-terminal probrain natriuretic peptide [3].

The nitric oxide pathway mediates its action through increasing intracellular cyclic GMP levels [39]. This can be enhanced by PDEVi such as sildenafil and tadalafil, of benefit in patients with CHD-APAH and it is very cheap to administer [3]. Moreover, together with nitric oxide, sildenafil has been shown to be beneficial in the treatment of pulmonary hypertensive crises after cardiothoracic surgery [3]. Sildenafil, however, needs to be given every 8 hours, which can be inconvenient, so the alternative tadalafil has been introduced, which has the advantage of daily administration [40]. Riociguat has been recently introduced as a soluble guanylate cyclase stimulator and as such, is only in standard use for chronic thromboembolic pulmonary hypertension that is inoperable or that persists after pulmonary endarterectomy [41]. It has, however, now increasingly been introduced for patients with class $1 \mathrm{PAH}$ as shown in the PATENT and RESPITE studies [42,43].

Endothelin receptor antagonists such as bosentan, macitentan, and ambrisentan have also demonstrated favorable results when used in patients with CHD-APAH [3].

Bosentan, a dual-receptor endothelin antagonist, was found to improve the 6-min walk test and decrease PVR after 16 weeks in patients with Eisenmenger [7]. It reduces PAP and attenuated pulmonary fibrosis and inflammation in animal studies. Uncontrolled studies have shown positive results in pediatric patients, similar to that of adults, with survival rates around $80-90 \%$ at 1 year [3]. Therefore, Bosentan is an increasingly used therapy in symptomatic patients with CHD-APAH. Ambrisentan is relatively $\mathrm{ET}_{\mathrm{A}}$ selective and has few drug-drug interactions. Ongoing discussion ensues as to whether this is a preferred option - or whether the enhanced dual receptor antagonist Macitentan is preferred in CHD-APAH. Macitentan has slow dissociation kinetics, has high receptor occupancy half-life and is the first in class to provide noncompetitive antagonism at the endothelin receptor [44-46].

The final class of disease modifying therapy used in CHD-APAH, is the prostanoid group such as epoprostenol and iloprost. Prostacyclin analogs (epoprostenol) have been shown to improve pulmonary hemodynamics, oxygen saturations and functional capacity in patients with CHD-APAH [3]. Epoprostenol is used in patients with repaired CHD but the use of central lines exposes the patient to sepsis as well as paradoxical embolism in those with unrepaired defects [47]. Therefore, oral or inhaled therapies are preferred in CHD as opposed to intravenous therapies. Iloprost is an inhaled synthetic prostacyclin analogue and subcutaneous treprostinil are is very effective. However, the presence of side effects or facial vasodilation and flushing, as well as the inconvenience of twice-hourly inhalations or painful injections throughout the day, limits their use [48].

Selexipag, a prostacyclin receptor agonist, has only recently been licensed so its use is likely to increase in these patients. At the moment, the role for this promising therapy is not yet clarified in CHD. 
Surgical options are also available including ductal stenting for persistent ductus arteriosus, surgical Potts shunt and atrial septostomy $[49,50]$. In addition, lung transplantation remains an option for patients with CHD-APAH. However, the number of donors is very small. Often the patients with CHD-APAH are too well to be placed on the transplant list, until they deteriorate suddenly when they become too sick. In practical terms, lung transplantation is not usually an option for these patients.

\section{Outcomes}

With earlier surgery to repair cardiac defects, outcomes have improved over the last two decades. However, there are still challenges raised with the complications associated with pulmonary hypertension in those unrepaired or only palliated. These include hyperviscosity symptoms, abnormal hemostasis, hemoptysis, intrapulmonary thrombosis and infections [21]. Chronic cyanosis causes increased erythropoietin with subsequent erythropoiesis and secondary erythrocytosis. This ensures adequate tissue oxygenation, preventing hypoxic end-organ damage. There have been some associations between increased hematocrit and hyperviscosity symptoms such as headaches, dizziness, visual disturbances, paraesthesia and myalgia. However, this link remains inconclusive. In addition, chronic cyanosis is associated with thrombocytopenia and platelet dysfunction with abnormalities in the clotting cascade [51]. Thus, abnormal hemostasis and bleeding problems are common in Eisenmenger patients. Furthermore, data shows intrapulmonary thrombosis in up to a third of adults with Eisenmenger especially in those with large ASDs [52]. This can cause pulmonary infarction when left untreated.

Infections, namely bacterial endocarditis, pose a huge risk to any patient with CHD, with unrepaired posing a bigger threat [21]. Data shows the lifetime risk of developing endocarditis in a patient with an unrepaired VSD is up to $13 \%$ [53]. This can also increase the risk of septic emboli and cerebral abscesses [21]. Therefore, prophylaxis against bacterial endocarditis is needed in patients with Eisenmenger syndrome [54].

Last, arrhythmias are frequent late sequelae in Eisenmenger syndrome, leading to heart failure and occasionally sudden cardiac death. It has been shown that up to $42 \%$ of patients with Eisenmenger have supraventricular arrhythmias on routine ECG and 24-holter monitoring during follow up [55].

The mortality in CHD-PAH is not fully characterized. This is partly due to the lack of registries for such patients in most countries, especially in the UK. However, it is recognized that pulmonary vasodilator therapies are associated with greater survival in Eisenmenger patients. The risk of death was $8.4 \%$ per year in those not on therapy but $4.8 \%$ per year in those who have been commenced on one of the therapies described above [56]. This is the encouragement that is needed for those caring for such patients to ensure the best possible care and early therapy if appropriate.

\section{Future perspective}

With improvements in morbidity and mortality from early surgical intervention, it can be anticipated that through continuing research and education, earlier detection and intervention of CHD-APAH may be expected. However, whether this will decrease the prevalence of the condition is uncertain. Ongoing trials into the treatment of $\mathrm{PH}$, and particularly in patients with CHD, may give rise to improved survival in the future. For example, a new dual receptor antagonist, macitentan, is currently undergoing trials to determine its efficacy in simple Eisenmenger syndrome [57]. This is mostly well tolerated with encouraging signs in relation to oxygen saturations and efficacy. Furthermore, the pathophysiology of the effects on lung function is still unclear and future research can help to provide clearer understandings of disease processes in $\mathrm{PH}$ associated with $\mathrm{CHD}$ and hence guide potential treatments [58].

Financial \& competing interests disclosure

The authors have no relevant affiliations or financial involvement with any organization or entity with a financial interest in or financial conflict with the subject matter or materials discussed in the manuscript. This includes employment, consultancies, honoraria, stock ownership or options, expert testimony, grants or patents received or pending, or royalties.

No writing assistance was utilized in the production of this manuscript.

Open access

This work is licensed under the Attribution-NonCommercial-NoDerivatives 4.0 Unported License. To view a copy of this license, visit http://creativecommons.org/licenses/by-nc-nd/4.0/〈0:headerinfo_indesign $\rangle\langle 0$ :clearpage $\rangle\langle/ 0$ :clearpage $\rangle\langle 0$ :anchor $\rangle$ BB1 $\langle/ 0$ :an chor $\rangle / / 0$ :headerinfo_indesign $\rangle$ 


\section{Executive summary}

\section{Definitions}

- Pulmonary hypertension is defined as an increase in mean pulmonary arterial pressure $\geq 25 \mathrm{mmHg}$, measured during right heart catheterization at rest.

Epidemiology

- There is a prevalence of congenital heart disease associated pulmonary artery hypertension (CHD-APAH) of $5-10 \%$ in adults with congenital heart disease.

Causes \& pathophysiology

- CHD-APAH is commonly secondary to left-to-right shunt defects or left heart obstructive disease causing postcapillary hypertension but is often multifactorial.

- Atrial connections, even with large defects, develop Eisenmenger much later in life than defects at other levels.

Presentation \& diagnosis

- Symptoms of pulmonary hypertension (PH) include shortness of breath, fatigue, anemia and syncope.

- If pulmonary artery hypertension is advanced, more severe signs relating to right heart failure can be seen.

- Exercise testing is done with either a 6-min walk test or cardiopulmonary exercise testing with measurement of peak oxygen consumption.

- Blood tests include full blood count, urea and electrolytes, liver function tests and uric acid.

- Echocardiography visualizes anatomy and doppler measurements can be used to estimate pulmonary arterial pressure and look for other signs of $\mathrm{PH}$.

- Right heart catheter is often required to confirm the diagnosis of CHD-APAH.

- In those patients with the Fontan circulation, accurate measurement of pulmonary artery pressure, transpulmonary gradient and pulmonary vascular resistance is mandatory before starting therapy.

- All CT scans in cardiac centers are now high resolution and this is the modality of choice for assessing lung parenchyma, especially to exclude intrapulmonary hemorrhage or infarction.

- MRI scans can add further information about right ventricle function or hybrid measurements of pulmonary vascular resistance.

Treatment

- Standard therapy involves diuretics, with oxygen therapy of limited use.

- Calcium channel blockers are negatively inotropic and hence not recommended.

- Therapy involves use of phosphodiesterase inhibitors (sildenafil and tadalafil), endothelin receptor blockades (bosentan, macitentan and ambrisentan) and synthetic prostacyclins or prostacyclin receptor (IP) stimulation (epoprostenol and iloprost).

- Surgical options may also available including ductal stenting for Persistent Ductus Arteriosus (PDA), surgical potts shunt and atrial septostomy.

Outcomes

- With earlier surgery to repair cardiac defects, outcomes have improved over the last two decades.

- However, there are still challenges raised with the complications associated with pulmonary hypertension especially in those with unrepaired or partially repaired defects.

- Last, arrhythmias are frequent late sequelae in Eisenmenger syndrome, leading to heart failure and occasionally sudden cardiac death.

Future perspective

- Ongoing drug trials may give potential new therapies in the future.

- However, further research is still needed to better understand the disease processes in $\mathrm{PH}$, and in particular, that is related to congenital heart disease.

- As of yet, we do not know which therapy is best for CHD-APAH, whether disease modifying therapy is appropriate in segmental pulmonary artery hypertension and what the long-term outcome holds for these patients.

- It is clear that attempted repair of defects in patients with Eisenmenger might accelerate the disease process.

\section{References}

Papers of special note have been highlighted as: $\bullet$ of interest

1. Hoeper MM. Definition, classification, and epidemiology of pulmonary arterial hypertension. Semin. Respir. Crit. Care Med. 30(4), 369-375 (2009).

2. Vachiery JL, Adir Y, Barbera JA et al. Pulmonary hypertension due to left heart diseases. J. Am. Coll. Cardiol. 62(Suppl. 25), D100-D108 (2013). 
3. Galie N, Humbert M, Vachiery JL et al. 2015 ESC/ERS Guidelines for the diagnosis and treatment of pulmonary hypertension: the joint task force for the Diagnosis and Treatment of Pulmonary Hypertension of the European Society of Cardiology (ESC) and the European Respiratory Society (ERS): endorsed by: Association for European Paediatric and Congenital Cardiology (AEPC), International Society for Heart and Lung Transplantation (ISHLT). Eur. Respir. J. 37(1), 67-119 (2015).

- This is the up to date European guidelines for the management of pulmonary hypertension and is a key document guiding current management.

4. Diller GP, Dimopoulos K, Okonko D et al. Exercise intolerance in adult congenital heart disease: comparative severity, correlates, and prognostic implication. Circulation 112(6), 828-835 (2005).

5. Duffels MG, Engelfriet PM, Berger RM et al. Pulmonary arterial hypertension in congenital heart disease: an epidemiologic perspective from a Dutch registry. Int. J. Cardiol. 120(2), 198-204 (2007).

6. Engelfriet PM, Duffels MG, Moller T et al. Pulmonary arterial hypertension in adults born with a heart septal defect: the Euro Heart Survey on adult congenital heart disease. Heart 93(6), 682-687 (2007).

7. Farber HW, Loscalzo J. Pulmonary arterial hypertension. N. Engl. J. Med. 351(16), 1655-1665 (2004).

8. Roberts KE, Mcelroy JJ, Wong WP et al. BMPR2 mutations in pulmonary arterial hypertension with congenital heart disease. Eur. Respir. J. 24(3), 371-374 (2004).

9. Harrison RE, Berger R, Haworth SG et al. Transforming growth factor-beta receptor mutations and pulmonary arterial hypertension in childhood. Circulation 111(4), 435-441 (2005).

10. Diller GP, Kempny A, Inuzuka R et al. Survival prospects of treatment naive patients with Eisenmenger: a systematic review of the literature and report of own experience. Heart 100(17), 1366-1372 (2014).

- Gives details about treatment-naive patients and allows comparison with treated patients in the modern era.

11. Simonneau G, Galie N, Rubin LJ et al. Clinical classification of pulmonary hypertension. J. Am. Coll. Cardiol. 43(Suppl. 12), S5-S12 (2004).

12. Cossio-Aranda J, Zamora KD, Nanda NC et al. Echocardiographic correlates of severe pulmonary hypertension in adult patients with ostium secundum atrial septal defect. Echocardiography 33(12), 1891-1896 (2016).

13. Samaan HA. Surgery of ventricular septal defect and pulmonary vascular resistance. Thorax 25(6), 665-668 (1970).

14. De Vadder K, Van De Bruaene A, Gewillig M, Meyns B, Troost E, Budts W. Predicting outcome after Fontan palliation: a single-centre experience, using simple clinical variables. Acta Cardiol. 69(1), 7-14 (2014).

15. Gewillig M, Brown SC, Eyskens B et al. The Fontan circulation: who controls cardiac output? Interact. Cardiovasc. Thorac. Surg. 10(3), $428-433$ (2010).

- Gives important guidance to the understanding of the physiology of Fontan circulation.

16. Kozlik-Feldmann R, Hansmann G, Bonnet D, Schranz D, Apitz C, Michel-Behnke I. Pulmonary hypertension in children with congenital heart disease (PAH-CHD, PPHVD-CHD). Expert consensus statement on the diagnosis and treatment of paediatric pulmonary hypertension. The European Paediatric Pulmonary Vascular Disease Network, endorsed by ISHLT and DGPK. Heart 102(Suppl. 2), ii42-ii48 (2016).

17. Diller GP, Gatzoulis MA. Pulmonary vascular disease in adults with congenital heart disease. Circulation 115(8), 1039-1050 (2007).

18. Meyrick B, Reid L. Ultrastructural findings in lung biopsy material from children with congenital heart defects. Am. J. Pathol. 101, 527-537 (1980).

19. Rabinovitch M. Molecular pathogenesis of pulmonary arterial hypertension. J. Clin. Invest. 122(12), 4306-4313 (2012).

20. Iacobazzi D, Suleiman MS, Ghorbel M, George SJ, Caputo M, Tulloh RM. Cellular and molecular basis of RV hypertrophy in congenital heart disease. Heart 102(1), 12-17 (2016).

21. Bradford R, Tulloh R. Diagnosis and management of pulmonary hypertension in adult congenital heart disease. Br. J. Cardiac Nurs. 3 , $138-145$ (2008).

22. Rubens $\mathrm{C}$, Ewert R, Halank M et al. Big endothelin-1 and endothelin-1 plasma levels are correlated with the severity of primary pulmonary hypertension. Chest 120(5), 1562-1569 (2001).

23. Badesch DB, Mclaughlin VV, Delcroix M et al. Prostanoid therapy for pulmonary arterial hypertension. J. Am. Coll. Cardiol. 43(Suppl. 12), S56-S61 (2004).

24. Christman BW, Mcpherson CD, Newman JH et al. An imbalance between the excretion of thromboxane and prostacyclin metabolites in pulmonary hypertension. N. Engl. J. Med. 327(2), 70-75 (1992).

25. Breuer J, Georgaraki A, Sieverding L, Baden W, Apitz J. Increased turnover of serotonin in children with pulmonary hypertension secondary to congenital heart disease. Pediatr. Cardiol. 17(4), 214-219 (1996).

26. Cantor WJ, Harrison DA, Moussadji JS et al. Determinants of survival and length of survival in adults with Eisenmenger syndrome. Am. J. Cardiol. 84(6), 677-681 (1999).

27. Bonderman D, Wexberg P, Martischnig AM et al. A noninvasive algorithm to exclude pre-capillary pulmonary hypertension. Eur. Respir. J. 37(5), 1096-1103 (2011). 
28. Paciocco G, Martinez FJ, Bossone E, Pielsticker E, Gillespie B, Rubenfire M. Oxygen desaturation on the six-minute walk test and mortality in untreated primary pulmonary hypertension. Eur. Respir. J. 17(4), 647-652 (2001).

29. Miyamoto S, Nagaya N, Satoh T et al. Clinical correlates and prognostic significance of six-minute walk test in patients with primary pulmonary hypertension. Comparison with cardiopulmonary exercise testing. Am. J. Respir. Crit. Care Med. 161(2 Pt 1), 487-492 (2000).

30. Van De Bruaene A, De Meester P, Voigt JU et al. Worsening in oxygen saturation and exercise capacity predict adverse outcome in patients with Eisenmenger syndrome. Int. J. Cardiol. 168(2), 1386-1392 (2013).

31. Kaemmerer HFS, Braun SL, Koelling $\mathrm{K}$ et al. Erythrocyte indexes, iron metabolism and hyperhomocysteinemia in adults with cysnotic congenital cardiac disease. Am. J. Cardiol. 94, 825-828 (2004).

- A useful source of information about the role of iron and why we keep our patients iron-replete even though their hemoglobin levels are higher than in normal patients in the presence of cyanotic congenital heart disease.

32. Van De Bruaene A, Delcroix M, Pasquet A et al. Iron deficiency is associated with adverse outcome in Eisenmenger patients. Eur. Heart J. 32(22), 2790-2799 (2011).

33. D'alto M, Dimopoulos $\mathrm{K}$, Budts W et al. Multimodality imaging in congenital heart disease-related pulmonary arterial hypertension. Heart 102(12), 910-918 (2016).

- Useful information about the diagnostic tools for imaging in congenital heart disease.

34. Post MC, Janssens S, Van De Werf F, Budts W. Responsiveness to inhaled nitric oxide is a predictor for mid-term survival in adult patients with congenital heart defects and pulmonary arterial hypertension. Eur. Heart J. 25(18), 1651-1656 (2004).

35. Peacock AJ, Crawley S, Mclure $\mathrm{L}$ et al. Changes in right ventricular function measured by cardiac magnetic resonance imaging in patients receiving pulmonary arterial hypertension-targeted therapy: the EURO-MR study. Circ. Cardiovasc. Imaging 7(1), 107-114 (2014).

36. Swift AJ, Rajaram S, Condliffe R et al. Diagnostic accuracy of cardiovascular magnetic resonance imaging of right ventricular morphology and function in the assessment of suspected pulmonary hypertension results from the ASPIRE registry. J. Cardiovasc. Magn. Reson. 14, 40 (2012).

37. Shujaat A, Bajwa AA, Al-Saffar F, Bellardini J, Jones L, Cury JD. Diagnostic accuracy of echocardiography combined with chest CT in pulmonary hypertension. Clin. Respir. J. 12(3), 948-952 (2017).

38. Sandoval J, Aguirre JS, Pulido T et al. Nocturnal oxygen therapy in patients with the Eisenmenger syndrome. Am. J. Respir. Crit. Care Med. 164(9), 1682-1687 (2001).

39. Moncada S. Nitric oxide. J. Hypertens. Suppl. 12(10), S35-S39 (1994).

40. Galie N, Brundage BH, Ghofrani HA et al. Tadalafil therapy for pulmonary arterial hypertension. Circulation 119(22), 2894-2903 (2009).

41. Ghofrani HA, D'armini AM, Grimminger F et al. Riociguat for the treatment of chronic thromboembolic pulmonary hypertension. $N$. Engl. J. Med. 369(4), 319-329 (2013).

42. Ghofrani HA, Galie N, Grimminger F et al. Riociguat for the treatment of pulmonary arterial hypertension. N. Engl. J. Med. 369(4), 330-340 (2013).

43. Hoeper MM, Klinger JR, Benza RL et al. Rationale and study design of RESPITE: an open-label, Phase IIIB study of riociguat in patients with pulmonary arterial hypertension who demonstrate an insufficient response to treatment with phosphodiesterase-5 inhibitors. Respir. Med. 122(Suppl. 1), S18-S22 (2017).

44. Iglarz M, Binkert $\mathrm{C}$, Morrison $\mathrm{K}$ et al. Pharmacology of macitentan, an orally active tissue-targeting dual endothelin receptor antagonist. J. Pharmacol. Exp. Ther. 327(3), 736-745 (2008).

45. Iglarz M, Bossu A, Wanner $\mathrm{D}$ et al. Comparison of pharmacological activity of macitentan and bosentan in preclinical models of systemic and pulmonary hypertension. Life Sci. 118(2), 333-339 (2014).

46. Iglarz M, Landskroner $\mathrm{K}$, Bauer $\mathrm{Y}$ et al. Comparison of macitentan and bosentan on right ventricular remodeling in a rat model of non-vasoreactive pulmonary hypertension. J. Cardiovasc. Pharmacol. 66(5), 457-467 (2015).

47. Rosenzweig EB, Kerstein D, Barst RJ. Long-term prostacyclin for pulmonary hypertension with associated congenital heart defects. Circulation 99(14), 1858-1865 (1999).

48. Hoeper MM, Leuchte H, Halank M et al. Combining inhaled iloprost with bosentan in patients with idiopathic pulmonary arterial hypertension. Eur. Respir. J. 28(4), 691-694 (2006).

49. Boudjemline Y, Patel M, Malekzadeh-Milani S, Szezepanski I, Levy M, Bonnet D. Patent ductus arteriosus stenting (transcatheter Potts shunt) for palliation of suprasystemic pulmonary arterial hypertension: a case series. Circ. Cardiovasc. Interv. 6(2), e18-e20 (2013).

50. Baruteau AE, Belli E, Boudjemline Y et al. Palliative Potts shunt for the treatment of children with drug-refractory pulmonary arterial hypertension: updated data from the first 24 patients. Eur. J. Cardiothorac. Surg. 47(3), e105-e110 (2015).

51. Humbert M, Morrell NW, Archer SL et al. Cellular and molecular pathobiology of pulmonary arterial hypertension. J. Am. Coll. Cardiol. 43(12 Suppl.), S13-S24 (2004).

- A good clear description of the molecular pathobiology of pulmonary hypertension. 
52. Broberg C, Ujita M, Babu-Narayan S et al. Massive pulmonary artery thrombosis with haemoptysis in adults with Eisenmenger's syndrome: a clinical dilemma. Heart 90(11), e63 (2004).

53. Shah P, Walter S, Rose V, Keith JD. Incidence of bacterial endocarditis in ventricular septal defects. Circulation 34, 127-131 (1966).

54. Dajani AS, Taubert K, Wilson W, Bolger AF et al. Prevention of bacterial endocarditis: recommendations by the American Heart Association. Circulation 96, 358-366 (1997).

55. Daliento L, Somerville J, Presbitero P, Menti L, Brach-Prever S, Rizzoli G, Stone S. Eisenmenger syndrome: factors relating to deterioration and death. Eur. Heart J. 19, 1845-1855 (1998).

- Although an old paper, it gives predictors for outcome in Eisenmenger syndrome.

56. Arnott C, Strange G, Bullock A et al. Pulmonary vasodilator therapy is associated with greater survival in Eisenmenger syndrome. Heart doi:10.1136/heartjnl-2017-311876 (2017) (Epub ahead of print).

57. Herbert S, Gin-Sing W, Howard L, Tulloh RM. Early experience of Macitentan for pulmonary arterial hypertension in adult congenital heart disease. Heart Lung Circ. 26(10), 1113-1116 (2017).

- This is a key paper in describing the first experience of macitentan in congenital heart disease before the results of the MAESTRO study are published.

58. Low AT, Medford AR, Millar AB, Tulloh RM. Lung function in pulmonary hypertension. Respir. Med. 109(10), 1244-1249 (2015).

59. Galiè N, Torbicki A, Barst R et al. Guidelines on diagnosis and treatment of pulmonary arterial hypertension: the Task Force on Diagnosis and Treatment of Pulmonary Arterial Hypertension of the European Society of Cardiology. Eur. Heart J. 25(24), 2243-2278 (2004). 
